\title{
Plasmon-Enhanced Second-Harmonic Generation from lonic Self-Assembled Multilayer Films
}

2007

Vol. 7, No. 2

254-258

\author{
Kai Chen, Cemil Durak, J. R. Heflin, and Hans D. Robinson* \\ Department of Physics, Virginia Tech, Blacksburg, Virginia 24061
}

Received September 5, 2006; Revised Manuscript Received November 20, 2006

\begin{abstract}
We have demonstrated large enhancements of the effective second-order nonlinear susceptibility $\left(\chi^{(2)}\right)$ of ionic self-assembled multilayer (ISAM) films, causing a film with just 3 bilayers to be optically equivalent to a 700-1000 bilayer film. This was accomplished by using nanosphere lithography to deposit silver nanoparticles on the ISAM film, tuning the geometry of the particles to make their plasmonic resonances overlap the frequency of optical excitation. An enhancement in the efficiency of second harmonic generation (SHG) by as much as 1600 times was observed. Even though this is already a large value, we suggest that further refinements of the techniques are expected to lead to additional enhancements of similar or larger magnitude.
\end{abstract}

Second-order nonlinear optical (NLO) materials are at the heart of telecommunications devices such as electro-optic modulators and optical switches, and in lasers such as highpower green and blue solid-state lasers and optical parametric amplifiers.

Conventional NLO materials generally consist of inorganic crystals such as KTP, $\mathrm{LiTaO}_{3}$, and $\mathrm{LiNbO}_{3}{ }^{1}{ }^{1}$ While they are quite efficient, high-quality crystals of sufficient size are expensive and difficult to manufacture. Organic NLO materials provide an alternative with the potential to provide high nonlinear susceptibilities in an economical fabrication process. ${ }^{2}$ NLO materials based on ionic self-assembled multilayer (ISAM) films are particularly promising because of the ease of tailoring noncentrosymmetric structures and the long-term stability. ${ }^{3-5}$ These films are made by alternately immersing a substrate in two solutions, containing a polycation and a polyanion, respectively. If the substrate initially carries negative surface charges, dipping in the polycation solution will result in a nanoscale polymer layer selfassembled on the substrate, yielding a positively charged substrate. Subsequent dipping in the polyanion solution results in a second layer of the polyanion formed on top of the first layer. The process can be repeated as many times as desired, building up films to arbitrary thickness with nanoscale precision. ISAM films may have substantial $\chi^{(2)}$ values, comparable to that of lithium niobate. ${ }^{5}$ Various methods have been suggested to improve the effective $\chi^{(2)}$ of these films by modifying their composition. ${ }^{6,7}$ Here, we demonstrate a new approach to enhance the second-order NLO susceptibility by creating hybrid structures from ISAM films and noble metal nanoparticles.

* To whom correspondence should be addressed. E-mail: hansr@ vt.edu.
Nanoparticles made from noble metals such as silver or gold have recently attracted considerable attention due to their unusual optical properties which enable light to be controlled in unique new ways..$^{8-10}$ The interaction of light with the free electrons in such particles gives rise to collective oscillations of the conduction electrons at optical frequencies, known as localized surface plasmons resonances (LSPRs). When excited in this fashion, the particles act as nanoscale antennas, concentrating the electromagnetic (E-M) field into very small volumes adjacent to the particles. Exceptionally large enhancements in E-M intensity can be obtained this way, as much as a factor of $10^{4}$ in individual particles and $10^{5}$ in dimers. ${ }^{11}$ Enhancements as large as $10^{7}$ have been reported in nanoparticle clusters, enabling, for example, Raman spectroscopy of single molecules. ${ }^{12,13}$

Other than Raman spectroscopy, ${ }^{14-16}$ this phenomenon has found applications in optical third-harmonic generation, ${ }^{17-19}$ as well as in second-harmonic generation, ${ }^{20-26}$ which is the subject of this paper. Nahata et al. ${ }^{23}$ demonstrated an $\sim 10^{4}$ fold increase in the efficiency of optical second-harmonic generation from concentric silver ring structures centered around a 200-nm aperture (bull's-eye structure) compared to a silver film with an unadorned aperture. Podlipensky et al. ${ }^{24}$ observed second harmonic generation (SHG) enhancement from ellipsoidal silver nanoparticles in a glass matrix grown by means of $\mathrm{Ag}^{+}-\mathrm{Na}^{+}$ion exchange. More recently, Moran et al. ${ }^{25}$ have demonstrated second harmonic excitation spectroscopy of silver nanoparticle arrays, which were fabricated by nanosphere lithography. In that work, a weak out-of-plane LSPR mode was made to overlap with the second harmonic of the incident laser, so that the resulting SHG emission signal became proportional to the plasmon 


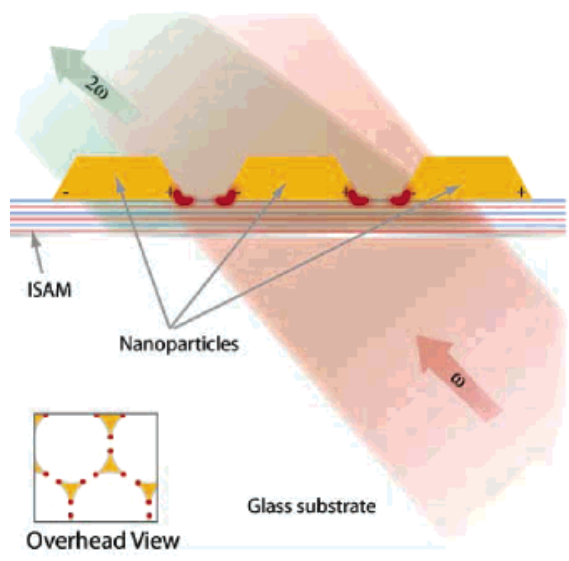

(a)

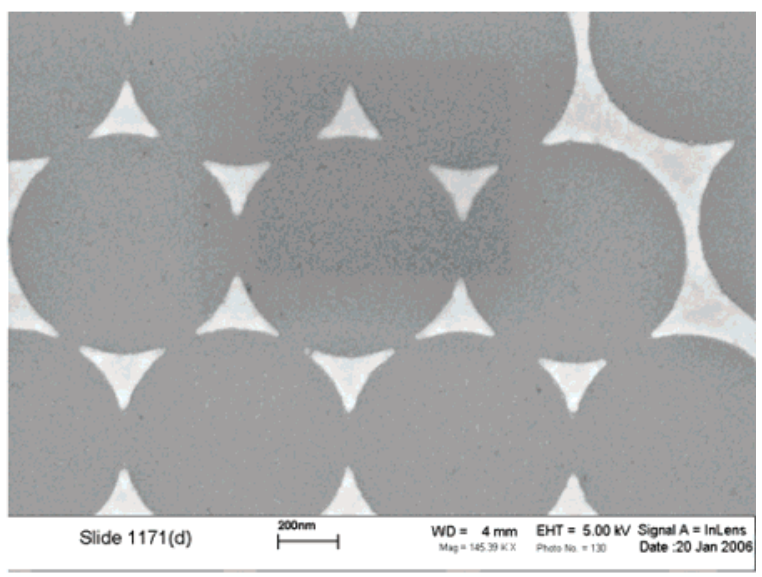

(b)

Figure 1. (a) Schematic sideview of an ISAM + metal nanoparticle hybrid film. A beam of light (red) traverses a layer of nanoparticles in the shape of triangular prismns (yellow). The localized intensity enhancement due to the LSPRs (red spots) leads to a commensurate increase in SHG light (green) from the ISAM film (red/white/blue). Inset: topview of the film. (b) SEM image of typical array of silver nanoparticles (bright phase) fabricated with nanosphere lithography, using spheres $720 \mathrm{~nm}$ in diameter as templates (removed). Note that the pattern is the same as that seen in the inset in (a).

enhancement. All of these studies used the nanoparticles in a dual role-as concentrators of the electromagnetic field and as the NLO media. Since the $\chi^{(2)}$ values of noble metals are very small, this gives rise to only modest nonlinear effects. In order to maximize the NLO efficiency, a better approach would be to combine the nanoparticles with a different material with an already strong NLO coefficient, as was envisioned by Pendry et al. ${ }^{27}$ Previous studies have used this approach to achieve high third-order NLO susceptibilities, where simple mixing of the two components is sufficient to obtain the enhancement. ${ }^{18,28}$ It is more difficult to implement this idea for second-order NLO effects, since the material is required to lack global inversion symmetry, which therefore means that a random mixture will have $\chi^{(2)}=0$.

In this paper, we demonstrate a dramatic increase in $\chi^{(2)}$ using plasmonic enhancement of organic NLO materials, where we have taken special care to ensure inversion asymmetry. Only one previous example of this is detailed in literature, ${ }^{29}$ in which cellular membranes were used to provide a weakly anisotropic environment for embedded metal nanoparticles coated with NLO dyes, leading to a moderate (20-fold) increase in SHG conversion efficiency. By contrast, our approach uses the inherently strong asymmetry of ISAM films to extract maximum advantage of the field concentrating properties of the LSPRs, as schematically shown in Figure 1a.

Standard glass microscope slides were used as substrates in the experiments. These slides were cleaned using the RCA cleaning process and then stored in deionized water until used. For the ISAM fabrication, PAH (poly(alylamine hydrochloride) $(10 \mathrm{mM} ; \mathrm{pH}=7)$ was used as the polycation and PCBS (poly(carboxyhydroxyphenylazobenzenesulfonamidoethanediyl) sodium salt) $(10 \mathrm{mM} ; \mathrm{pH}=7)$ as the polyanion. ISAM films were built up on the slides by alternately dipping them in these two solutions, taking care to rinse them thoroughly in deionized (DI) water between immersions. This naturally results in ISAM films on both sides of the slide. In the rest of this paper ISAM film thickness always refers to the thickness of the film on one side of the slide, even though both sides may be probed simultaneously.

Arrays of silver nanoparticles were fabricated on one side of the substrates, which were either bare or first coated with NLO ISAM films. Nanosphere lithography (NSL) ${ }^{30}$ was used in this step, because it allows rapid and simple deposition of relatively uniform nanoparticles. In this technique, a dispersion of polystyrene nanospheres is drop-cast onto the substrates (in our case consisting of 3.8\% $720 \mathrm{~nm}$ diameter spheres in DI water) and then allowed to dry. This causes the spheres to self-assemble into a close-packed monolayer. Silver is subsequently deposited onto the substrate using electron beam evaporation, after which the spheres are removed from the substrate by ultrasonication in dichloromethane for about $10 \mathrm{~s}$. Our tests indicate that the nonlinear properties of any underlying ISAM film are only marginally affected by this removal process. Since the deposited metal reaches the substrate only through the gaps in the closepacked film, this results in a honeycomb array of silver nanoparticles as shown in the micrograph in Figure 1b. In our work, the lateral dimensions of the nanoparticles were kept constant by the consistent use of $720 \mathrm{~nm}$ diameter nanospheres in all samples, while the optical properties of the particles were tuned by varying the thickness of the deposited metal from 30 to $100 \mathrm{~nm}$.

The LSPRs of the nanoparticle arrays were characterized by measuring the extinction spectrum of light transmitted through the substrate. A tungsten lamp, producing white light incident on the sample with an intensity of $2.1 \mathrm{~mW} / \mathrm{cm}^{2}$ was used as excitation source. Typical extinction spectra of $50 \mathrm{~nm}$ thick silver nanoparticles deposited on a bare glass substrate are shown in Figure 2. Three resonance peaks (labeled 1, 2, and 3) are seen in the wavelength range 350$1080 \mathrm{~nm}$, as in Figure 2a. The intensities and positions of the peaks vary a little for different spots on the same sample, 


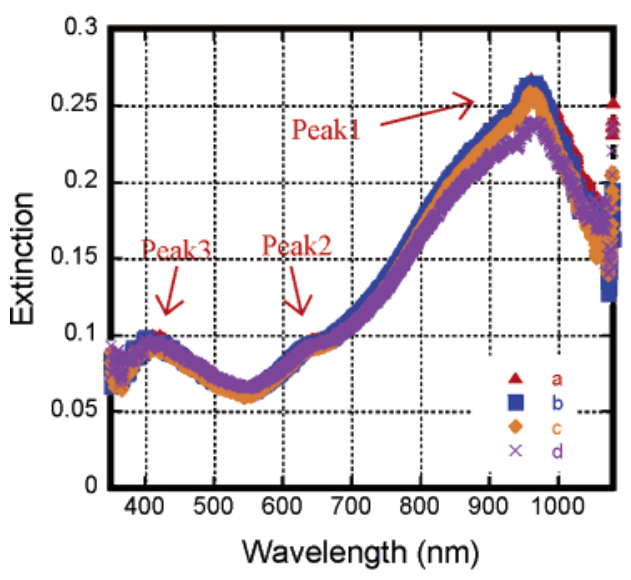

(a)

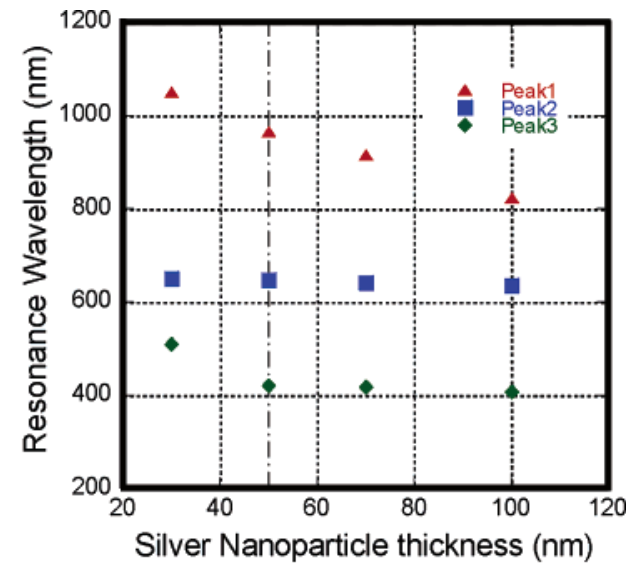

(b)

Figure 2. (a) Extinction spectra of $50 \mathrm{~nm}$ thick silver nanoparticle arrays fabricated using NSL on glass slides. Traces a-d are taken at different spots on the same substrate with a white light intensity of $2.1 \mathrm{~mW} / \mathrm{cm}^{2}$. Of the peaks indicated, peak 1 corresponds to the LSPR used for electromagnetic field enhancement. (b) Center wavelength of the plasmon resonance wavelength as a function of silver nanoparticle thickness. The tunability of peak 1 is exploited to tailor the operating wavelengths of the film. The data shown in (a) correspond to the dot-dashed line in (b).

which we ascribe to variations in the geometry of the nanoparticles across the substrate. Theoretical studies ${ }^{31,32}$ indicate that the three resonance bands can be attributed to the hybridization of dipole and quadruple modes, either in or out of the plane of the substrate. We tentatively ascribe the three peaks 1,2 , and 3 to out-of-plane dipole-like resonances, in-plane quadrupole-like resonances, and outof-plane quadrupole-like resonances, respectively. Figure $2 b$ shows the effect of the nanoparticle thickness on the resonance center wavelengths. It can be seen that peak 1, the dominant resonance, blue shifts strongly as the thickness increases, while the other two peaks depend only weakly on the particle thickness. This is in agreement with theoretical calculations for these triangle-shaped silver nanoparticles, ${ }^{31}$ as well as with previous experimental observations. ${ }^{33}$ This effect provides a means to tune the LSPR wavelength to the desired operating band. In this paper, we exploit it to make the dominant resonance (peak 1) overlap with the $1064 \mathrm{~nm}$ laser wavelength used in the SHG experiments described below.

Figure 3 illustrates the effect of depositing nanoparticles on ISAM films of varying thickness, rather than on a bare glass substrate. The presence of the ISAM film, which has a higher index of refraction $(\sim 1.6-1.7)$ than the glass $(\sim 1.5)$, causes a modulation of the LSPR wavelengths with ISAM thickness. This is reminiscent of result recently reported by Murray et al. ${ }^{34}$ and earlier by Holland and Hall..$^{35}$ In these works, the LSPR resonance frequency of metallic nanoparticles exhibited a similar modulation as a function of the distance to a nearby dielectric or metallic interface. The phenomenon was explained with a model by Chance, Prock, and Silbey, ${ }^{36}$ where the interference between the nanoparticles and their reflection off the interface leads to a periodic modulation in the resonance frequency as a function of the thickness of the dielectric spacer. In our case, the modulation takes place on a length scale $(\sim 10 \mathrm{~nm})$ that appears to be too short for this model to be applicable. It is therefore likely

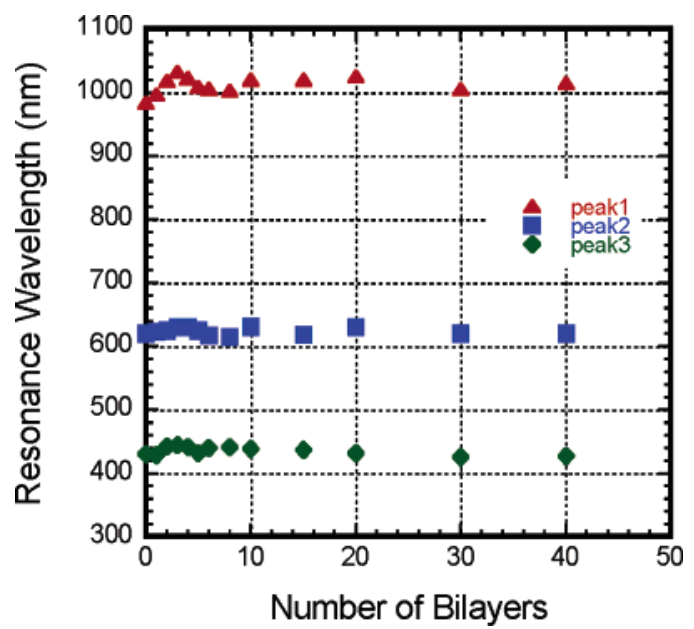

Figure 3. Center wavelengths of plasmon resonances in silver nanoparticles arrays fabricated using NSL on ISAM films of varying thickness. The data were obtained from transmission extinction spectra collected under $2.1 \mathrm{~mW} / \mathrm{cm}^{2}$ white light illumination. The nanoparticle thickness was $72 \mathrm{~nm}$ in all cases.

that a different model is needed to explain our data. However, this falls outside the scope of the present paper, and the LSPR peaks are sufficiently wide that this modulation can be ignored when choosing the nanoparticle thickness.

SHG was measured in a forward-transmission geometry using $11 \mathrm{~ns}$ long pulses from a Spectra-Physics Q-switched $10 \mathrm{~Hz} \mathrm{Nd:YAG,} \mathrm{with} \mathrm{a} \mathrm{fundamental} \mathrm{wavelength} \mathrm{of} 1064 \mathrm{~nm}$ as excitation source. The laser was incident on the sample with a spot $\sim 1 \mathrm{~mm}$ in diameter. The transmitted light was filtered to remove the laser wavelength, and the remaining SHG light was detected by a photomultiplier tube. The incident angle was varied by rotating of the samples with a computer-controlled rotation stage, and the incident intensity and polarization were controlled using a pair of Glan-Taylor polarizers. The samples were fabricated as described above, using $72 \mathrm{~nm}$ thick nanoparticles. This thickness ensures a sufficient overlap between laser emission and LSPR. 


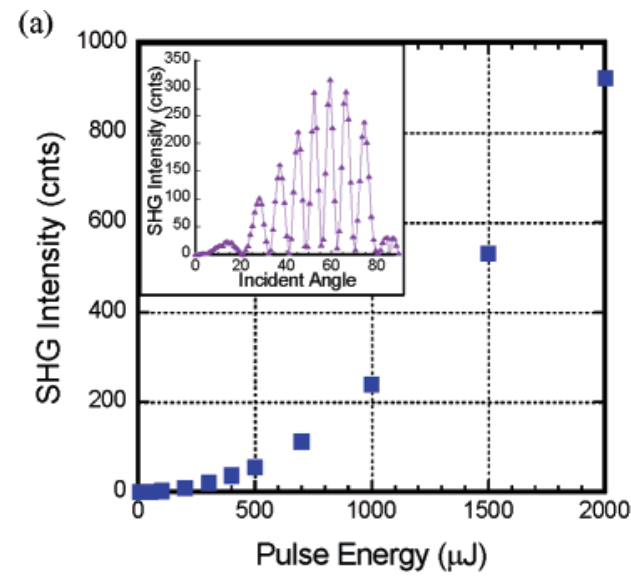

(b)

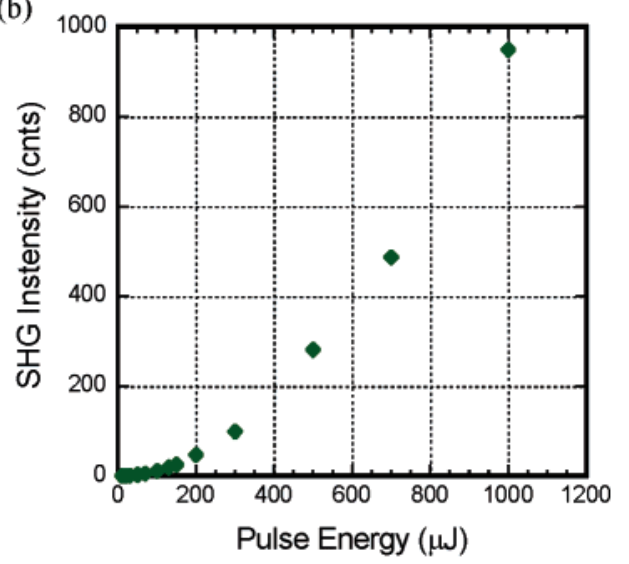

(c)

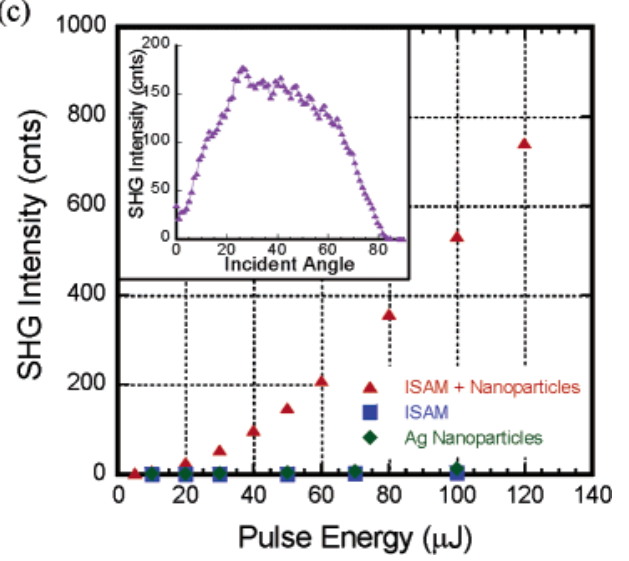

Figure 4. SHG signal obtained from (a) conventional 40 bilayer PAH/PCBS ISAM film, (b) nanoparticles deposited directly on a glass substrate, and (c) hybrid ISAM + nanoparticles films (red triangles) compared to the data from (a) and (b). The nanoparticle thickness was set to $72 \mathrm{~nm}$ to ensure a sufficient overlap between the laser excitation and the dominant LSPR. All data was taken close to a $45^{\circ}$ angle of incidence, adjusted in (a) to optimize phase matching between front and back films. The insets in (a) and (c) are Maker fringes from the conventional and hybrid films under 500 and $60 \mu \mathrm{J} /$ pulse excitation, respectively. In (a), varying phase matching between the front and back surface films generate a regular fringe pattern, while in (c), SHG from only the front side (containing the silver nanostructures) dominates, and the effect of the back side can be neglected.

Figure 4 illustrates the enhancement in SHG conversion efficiency brought about by combining NSL nanoparticles and a 40 bilayer NLO ISAM film. The $y$-axes in the three graphs are the same, so as to make the data easier to compare. In all three cases, the SHG intensity varies as the square of the excitation energy, as expected. All data were taken at an angle of incidence close to $45^{\circ}$, except for conventional ISAM films. In those cases, the angle was adjusted for optimum phase matching, which occurs around $46^{\circ}$, and the resulting signal was divided by 4 to remove the effect of the film on the reverse side of the substrate. The SHG conversion efficiency is similar for a conventional ISAM film alone (Figure 4a) and nanoparticles on a bare substrate alone (Figure 4b). However, when the two are combined (Figure 4c), a significantly lower excitation power is required to obtain the same SHG intensity. More precisely, the hybrid ISAM + nanoparticles film exhibits, in this case, over 200 times larger SHG conversion efficiency than either component alone. In fact, as the incident energy is increased in the hybrid ISAM + nanoparticles films, the sample burns, further illustrating the field-concentrating properties of the silver nanoparticles.

The insets in Figure 4 show Maker fringes ${ }^{37,38}$ (dependence of the SHG signal on incident angle) for the conventional films (in Figure 4a) and the hybrid films (Figure 4c). The former exhibits regular fringes with $100 \%$ visibility due to angle-dependent phase matching between the two identical films on either side of the substrate. The fringes are superimposed on a single peaked envelope that has the same functional form from one sample to the next. In the hybrid case, there are no regular fringes, because the nanoparticles are deposited only on one side of the substrate. Furthermore, the Maker fringes in theses samples are irregular, with shapes that vary between samples and between different spots on the same sample. As a result, SHG conversion efficiency for the hybrid films has an uncertainty of about a factor of 2. We believe that the likely origin of this irregularity lies in variations in the local orientation and quality of the nanoparticle arrays, so that the coupling of the laser to the LSPRs varies somewhat across the sample and as the incident angle is varied. A more systematic investigation of this phenomenon will be the subject of future work.

LSPR modes are likely to penetrate only a few nanometers into the ISAM film. Since each ISAM bilayer is approximately $1.2 \mathrm{~nm}$ thick, ${ }^{4}$ we can expect that the greatest enhancements in NLO properties will occur for ISAM films that are only a few bilayers thick. To test this hypothesis, we prepared conventional ISAM films with up to 300 bilayers as well as hybrid films with up to 40 bilayers and measured SHG conversion efficiency as described above. The results are shown in Figure 5. Focusing first on the conventional films (blue squares), one would naïvely expect the SHG conversion efficiency to grow quadratically with film thickness, which is not the case, except for the thickest films. The additional SHG signal seen for thin films can be attributed to interface effects. The polarizability of the top and bottom bilayers is substantially higher than for those in the bulk of the film, ${ }^{3}$ and a better model for the SHG intensity is given by $I_{2 \omega}=A[(N-2)+2 B]^{2} I_{\omega}{ }^{2}$, where $N$ is the number of bilayers and $A$ and $B$ are constants. The dashed line indicates a fit of this model to the data, with $A=7.06$ $\times 10^{-8}, B=8.59$. For the hybrid films, the SHG signal increases rapidly for the first three bilayers and then levels 


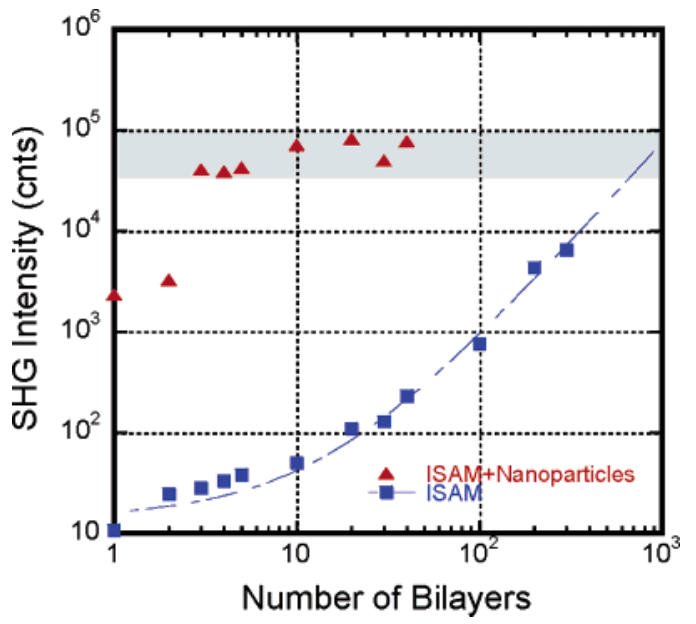

Figure 5. Comparison of SHG conversion efficiencies in conventional (blue squares) and hybrid (red triangles) ISAM films of varying thickness. The SHG intensity from the conventional film can be fitted to the model discussed in the text (dashed line), while the hybrid film SHG saturates at the level indicated by the gray band when the film becomes thicker than the decay length of the LSPR mode.

off to within the uncertainty due to the variations in nanoparticle quality and orientation discussed earlier (indicated by the gray band). This is precisely what we would expect, given that only the portion of the ISAM film that lies within the decay length of the most intense portion of the plasmon experiences significant field enhancement. We can then estimate this decay length to be of the order of the thickness of three ISAM bilayers, or $\sim 3-4 \mathrm{~nm}$. Because of this, the greatest enhancements in NLO coefficients are seen for the three bilayer film. At this thickness, the hybrid film generates 1600 times more SHG light than the conventional film, corresponding to an increase in $\chi^{(2)}$ of 40 times. Even more impressively, because the hybrid film takes maximum advantage of the interface enhancement, a conventional film would have to be $700-1000$ bilayers thick in order to be as efficient as the three bilayer hybrid film.

It is clear that the combination of plasmonically active nanoparticles with second-order NLO materials can be very fruitful. In this paper, we have investigated a very simple example of such a hybrid material where no efforts were made to optimize the structure of the particles or the film. We therefore anticipate that with more careful design of the films, further large improvements of the NLO properties are possible. This would, for instance, entail using metallic nanostructures with stronger E-M field enhancing properties and placing these more densely throughout the film. The resulting materials could have $\chi^{(2)}$ values well beyond those available today, which in turn would enable optical switches and modulators of smaller dimensions than what is currently available, while at the same time substantially reducing the cost of fabrication of electro-optic devices.

\section{References}

(1) Mizuuchi, K.; Yamamoto, K.; Kato, M.; Sato, H. IEEE J. Quantum Electron. 1994, 30 (7), 1596-1604.

(2) Endo, T.; Sueoka, K.; Mukasa, K. Jpn. J. Appl. Phys., Part 12000 , 39 (2A), 397-401.
(3) Van Cott, K. E.; Guzy, M. T.; Neyman, P. J.; Brands, C.; Heflin, J. R.; Gibson, H. W.; Davis, R. M. Angew. Chem., Int. Ed. 2002, 41, $3236-3238$.

(4) Heflin, J. R.; Figura, C.; Marciu, D.; Liu, Y.; Claus, R. O. Appl. Phys. Lett. 1999, 74 (4), 495-497.

(5) Heflin, J. R.; Guzy, M. T.; Neyman, P. J.; Gaskins, K. J.; Brands, C.; Wang, Z.; Gibson, H. W.; Davis, R. M.; VanCott, K. E. Langmuir 2006, 22 (13), 5723-5727.

(6) Neyman, P. J.; Shah, M. T. G. S.; Wang, H.; Gibson, H. W.; Van Cott, K. E.; Davis, R. M.; Figura, C.; Heflin, J. R. Polym. Mater. Sci. Eng. 2000, 83, 162-163.

(7) Neyman, P. J. M.S. Thesis, Virginia Polytechnic Institute and State University, 2002.

(8) Grigorenko, A. N.; Geim, A. K.; Gleeson, H. F.; Zhang, Y.; Firsov, A. A.; Khrushchev, I. Y.; Petrovic, J. Nature 2005, 438 (7066), 335338.

(9) Wang, H.; Brandl, D. W.; Le, F.; Nordlander, P.; Halas, N. J. Nano Lett. 2006, 6 (4), 827-832.

(10) Ebbesen, T. W.; Lezec, H. J.; Ghaemi, H. F.; Thio, T.; Wolff, P. A. Nature 1998, 391 (6668), 667-669.

(11) Hao, E.; Schatz, G. C. J. Chem. Phys. 2004, 120 (1), 357-366.

(12) Kneipp, K.; Wang, Y.; Kneipp, H.; Perelman, L. T.; Itzkan, I.; Dasari, R.; Feld, M. S. Phys. Rev. Lett. 1997, 78 (9), 1667-1670.

(13) Nie, S.; Emory, S. R. Science 1997, 275, 1102-1106.

(14) Fleischmann, M.; Hendra, P. J.; McQuillan, A. J. Chem. Phys. Lett. 1974, 26 (2), 163-166.

(15) Moskovits, M. Rev. Mod. Phys. 1985, 57 (3), 783.

(16) Baumberg, J. J.; Kelf, T. A.; Sugawara, Y.; Cintra, S.; Abdelsalam, M. E.; Bartlett, P. N.; Russell, A. E. Nano Lett. 2005, 5,(11), 22622267.

(17) Tsang, T. Y. F. Opt. Lett. 1996, 21 (4), 245-247.

(18) Dvir Yelin, D. O.; Thiberge, S.; Moses, E.; Silberber, Y. Opt. Express 2003, 11 (12), 1385-1391.

(19) Lippitz, M.; vanDijk, M. A.; Orrit, M. Nano Lett. 2005, 5 (4), 799802.

(20) Wokaun, A.; Bergman, J. G.; Heritage, J. P.; Glass, A. M.; Liao, P. F.; Olson, D. H. Phys. Rev. B 1981, 24 (2), 849.

(21) Boyd, G. T.; Rasing, T.; Leite, J. R. R.; Shen, Y. R. Phys. Rev. B 1984, 30 (2), 519.

(22) Muller, T.; Vaccaro, P. H.; Balzer, F.; Rubahn, H. G. Opt. Commun. 1997, 135 (1-3), 103-108.

(23) Ajay Nahata, R. A. L.; Ishi, T.; Ohashi, K. Opt. Lett. 2003, 28 (6), 423-425.

(24) Podlipensky, A.; Seifert, J. L. G.; Graener, H.; Cravetchi, I. Opt. Lett. 2003, 28 (9), 716-718.

(25) Moran, A. M.; Sung, J.; Hicks, E. M.; VanDuyne, R. P.; Spears, K. G. J. Phys. Chem. B 2005, 109 (10), 4501-4506.

(26) Fan, W.; Zhang, S.; Panoiu, N. C.; Abdenour, A.; Krishna, S.; Osgood, R. M.; Malloy, K. J.; Brueck, S. R. J. Nano Lett. 2006, 6 (5), 1027-1030.

(27) Pendry, J. B.; Holden, A. J.; Robbins, D. J.; Stewart, W. J. IEEE Trans. Microwave Theory Tech. 1999, 47 (11), 2075-2084.

(28) Shi-Wei, C.; Szu-Yu, C.; Tsung-Han, T.; Cheng-Chi, C.; Tzu-Ming, L.; Chi-Kuang, S.; Shr-Bin, W.; Sajal, B.; Juen-Kai, W.; Yu-Lin, W. In Functional THG microscopy with plasmon-resonance enhancement in silver nanoparticles; 2003; $\mathrm{p} 2$.

(29) Clark, H. A.; Campagnola, P. J.; Wuskell, J. P.; Lewis, A.; Loew, L. M. J. Am. Chem. Soc. 2000, 122 (41), 10234-10235.

(30) Haynes, C. L.; Van Duyne, R. P. J. Phys. Chem. B 2001, 105 (24), $5599-5611$.

(31) Jensen, T. R.; Duval, M. L.; Kelly, K. L.; Lazarides, A. A.; Schatz, G. C.; Van Duyne, R. P. J. Phys. Chem. B 1999, 103 (45), 98469853.

(32) Kelly, K. L.; Coronado, E.; Zhao, L. L.; Schatz, G. C. J. Phys. Chem. $B$ 2003, 107 (3), 668-677.

(33) Jensen, T. R.; Malinsky, M. D.; Haynes, C. L.; Van Duyne, R. P. J. Phys. Chem. B 2000, 104 (45), 10549-10556.

(34) Murray, W. A.; Suckling, J. R.; Barnes, W. L. Nano Lett. 2006, 6 (8), 1772-1777.

(35) Holland, W. R.; Hall, D. G. Phys. Rev. Lett. 1984, 52 (12), 1041.

(36) Chance, P. R.; Prock, A. P.; Silbey, R. Advanced Chem. Phys. 1978, $37,1-65$.

(37) Jerphagnon, J.; Kurtz, S. K. J. Appl. Phys. 1970, 41 (4), 1667-1681.

(38) Maker, P. D.; Terhune, R. W.; Nisenoff, M.; Savage, C. M. Phys. Rev. Lett. 1962, 8 (1), 21-23.

NL062090X 